Chimia 46 (1992) 33

(C) Schweiz. Chemiker-Verband; ISSN 0009-4293

\title{
Ecole d'ingénieurs ETS de Genève: diplôme d'ingénieur en génie chimique*
}

Les candidats au diplôme d'ingénieur en génie chimique se recrutent principalement à l'issue de la scolarité obligatoire: cycle d'orientation, section scientifique à Genève, études prégymnasiales vaudoises, préparations équivalentes des autres cantons (Tessin, Valais, etc.). Dans cette filière, Les études à l'EIG sont programmées en cinq ans, éventuellement en six ans pour les élèves qui ont besoin d'une année de préparation et suivent pour cela la classe dite 'de raccordement'.

Les personnes ayant suivi un apprentissage et qui sont au bénéfice d'un CFC, les porteurs d'une maturité ou d'un baccalauréat français, suivent une classe 'passerelle' qui leur permettra d'accomplir leurs études à l'EIG en quatre ans.

Cette option particulière de l'ecole d'ingénieurs de Genève, permet la formation d'ingénieurs-ETS dès la fin de la scolaritéobligatoire. Elle est possible grâce à la pratique integrée, ateliers d'apprentissage que suivent les élèves n'ayant pas de CFC, en parallèle avec la formation générale dispensée au cours des deux premières années.

Au cours des dix semestres généralement nécessaires à l'obtention du diplôme les études se répartissent de la manière suivante: les quatres premiers semestres de formation générale font partie d'un tronc commun pour les étudiants en génie mécanique et génie électrique. Cette première phase des études porte particulièrement sur: les mathématiques, l'informatique, la physique, le français, l'allemand et l'anglais, la chimie générale, la pratique intégrée.

A partir du 6ème semestre, l'enseignement comprend des disciplines propres à la formation de l'ingénieur, telles que la thermodynamique, la dynamique des fluides et la connaissance des matériaux. C'est également dès cette période que l'étudiant prendra connaissance des branches spécifiques à la profession: la planification et la construction des installations de production, tenant compte des aspects économiques, de sécurité et de l'environnement, l'étude des procédés mécaniques et thermiques, la chimie technique et l'étude de la conduite de réaction (procédés en chimie organique), la biotechnologie, la mesure et la régulation, l'informatique industrielle.
Les connaissances pratiques nécessaires pour la manipulation des produits et la conduite des appareils sont données dans divers laboratoires dont des laboratoires semi-industriels. Tout au long de ces 10 semestres d'étude, l'étudiant recoit les cours de culture générale (langues, communications) qui doivent lui permettre d'améliorer son mode d'expression.

Les études se terminent par un travail de diplôme réalisé sur une période limitée à 8 semaines, généralement en collaboration avec l'industrie et au cours desquels des problèmes spécifiques ont pu être résolus. L'étudiant devra alors livrer un mémoire et présenter son travail devant une commission d'experts provenant de l'industrie.

Les diplômés en génie chimique issus de notre école se trouvent plus particulièrement dans les secteurs de la planification, de la construction, dans la conduite des unités de production ainsi que dans le secteur technico-commercial.
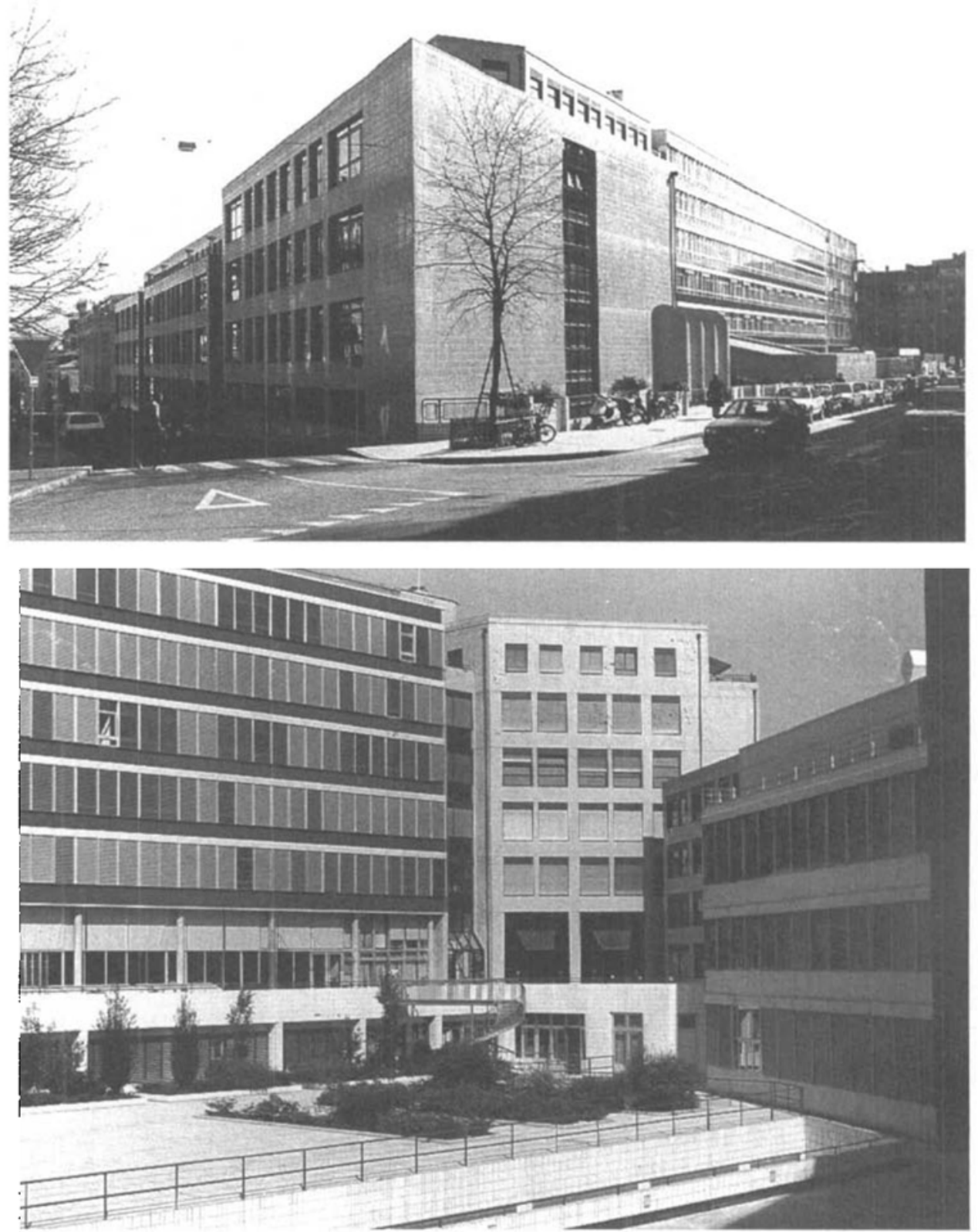